\title{
UN APAPAXTLI PARA CURAR EL ESTRÉS Y LA TRISTEZA. ESTUDIO DE UN CASO DE MEDICINA "TRADICIONAL" MEXICANA EN CIUDAD DE MÉXICO
}

\author{
AN APAPAXTLI TO CURE STRESS AND SADNESS. A CASE STUDY \\ OF MEXICAN FOLK MEDICINE IN MEXICO CITY
}

\author{
Nayelhi I. Saavedra Solano ${ }^{1}$ y Shoshana Berenzon Gorn ${ }^{2}$
}

\begin{abstract}
Presentamos las prácticas médicas de un grupo denominado Continua Tradición Tetzkatlipoka, cuyos saberes se atribuyen, principalmente, a los antiguos mexicanos. Sus actividades forman parte de la medicina "tradicional" mexicana y en consecuencia ellos son curanderos o médicos tradicionales. Estos curanderos tienen un repertorio de diferentes recursos y técnicas para tratar a las personas. Abordaremos una de éstas conocida como apapaxtli, vocablo náhuatl que significa "ablandar" y es utilizada por los curanderos para "suavizar" las emociones de las personas. Quienes recibieron el apapaxtli afirmaron que es un tratamiento doloroso pero efectivo. La investigación que presentamos fue realizada en Ciudad de México, sus objetivos fueron conocer las técnicas para tratar los padecimientos emocionales y analizar la experiencia que tuvieron algunas mujeres que recurrieron a esta medicina. Utilizamos la técnica de observación participativa y la de entrevista en profundidad al vocero oficial y a cinco de sus pacientes. Los saberes que estos médicos tienen acerca de la salud-enfermedad son consecuentes con sus postulados acerca del Universo y destacan el lugar privilegiado que ocupan las emociones. La relación médico-paciente que entablan propicia una atmósfera curativa benéfica para el paciente, pero sus efectos tienen escasa permanencia si el paciente no los prolonga con ayuda de otras técnicas que ofrece esta medicina.
\end{abstract}

Palabras claves: medicina tradicional mexicana, curanderos, padecimiento, usuarios.

In this paper we present the healing activities of the Continua Tradición Tetzkatlipoka group, whose knowledge is attributed to the ancient inhabitants of Mexico. Currently, such healing practices are grouped into the folk healing category and their practitioners are known as folk healers or medicine men. The folk healers we present here use different techniques and resources to cure people. We focus on just one technique, the apapaxtli, a nahuatl word that means "to soften". The data we present forms part of a larger research project in Mexico City. Through this study we sought to learn the methods and practices used to cure emotional problems and to explore the experiences of individuals who received these treatments. We conducted an in-depth interview of the group's spokesman group and five female patients. During the apapaxtli treatment we noticed a pleasant healer-patient relationship, which is beneficial for the patient. Nevertheless, we think that the benefits obtained by the patients could soon disappear if they do not follow the preventative measures recommended by these folk healers.

Key words: Mexican folk medicine, healers, illness, patients.

En Ciudad de México, como en otras ciudades de América Latina, es posible encontrar especialistas en distintas medicinas o maneras de curar, controlar y prevenir las enfermedades y malestares; entre ellas se encuentran la medicina tradicional china, el ayurveda, la homeopatía, el reiki y por supuesto la medicina científica (Luz 1999). Cada una de estas medicinas juega un papel específico dentro de la comunidad, ya sea como servicio de salud o como producción cultural.

En este trabajo hablaremos de la medicina conocida con la voz náhuatl wewepahtli ${ }^{1}$ (significa grandísima sanación) practicada por algunos miembros de la Continua Tradición Tetzkatlipoka², a quienes se les conoce como temaxtianitl (su significado es "el que lleva el sistema"). El grupo está adscrito a un colectivo heteróclito mayor conocido como "movimiento de mexicanidad" en el cual participan hombres y mujeres mestizos de diversas edades y condiciones socioeconómicas.

Ellos están plenamente integrados a la vida social urbana. Los miembros de la Tetzkatlipoka buscan mantener vigentes algunos aspectos culturales que atribuyen a los antiguos mexicanos y lo

1 Centro de Investigaciones y Estudios Superiores en Antropología Social. Juárez 22 Col Tlalpan C.P. 14000 México D.F. itandehuin@yahoo.com.mx

2 Instituto Nacional de Psiquiatría. Calzada México Xochimilco 101 Col San Lorenzo Huipulco Del. Tlalpan C.P. 14000 México D.F. berenz@imp.edu.mx 
hacen mediante la práctica de la danza, la música, la medicina, de los enlaces matrimoniales y en general un estilo de vida basado en la interpretación del repertorio simbólico perteneciente al grupo cultural nahua. La wewepahtli forma parte de la medicina "tradicional" mexicana; utilizamos este término para referirnos a un conjunto de conocimientos y prácticas generadas en el seno de la población. Estos saberes son de orden empírico y la mayoría de las veces se transmiten generacionalmente (Lozoya 1999). La fuente de inspiración de la wewepahtli procede de la cosmovisión de los pueblos nahuas, cosmovisión que ha sido interpretada por cada practicante a la vez que combinada con saberes provenientes de otros grupos culturales (de la población de origen africano, árabe, asiático) o inclusive con cuerpos teóricos como el de la biomedicina. La combinación a la que nos referimos resulta práctica común, ya que la medicina tradicional mexicana se encuentra sujeta a los cambios culturales y resulta más evidente en el medio urbano que en el rural. Un ejemplo de los cambios es la forma como se transmitían los conocimientos, comúnmente de padres o abuelos a hijos y nietos; ahora en Ciudad de México se han popularizado los cursos y talleres dirigidos a un amplio público. Aun con todos estos cambios no deja de reconocerse ni como tradicional ni como mexicana pues defiende su núcleo identitario mediante diferentes estrategias, recursos, denominaciones o estilos de atención que la distinguen de otras ofertas médicas.

Una de las hipótesis de trabajo fue que las ideas y creencias que cualquier grupo social sostiene acerca de la enfermedad, la salud, las maneras sobre cómo ésta se pierde y recupera guardan una estrecha correspondencia con sus valores morales, nociones de cuerpo, representaciones de belleza, entre otras elaboraciones intelectuales; además este repertorio simbólico fundamenta su orden social y favorece la reproducción de sus formas culturales (Augé y Herzlich 1994). Al mismo tiempo consideramos que el lenguaje interviene, necesariamente, para la producción de la materia simbólica pues no solamente es un instrumento de comunicación sino que es un sistema clasificatorio que determina cuáles son las percepciones potencialmente sociales (Hodge y Kress 1993) y permite la construcción de la realidad. Así que las nociones sobre el proceso salud-enfermedad que un individuo exprese siempre presentarán un marcaje que corresponderá con sus particularidades culturales y sociales. Con el objetivo de aprehender las experiencias y elaboraciones discursivas de las mujeres de la manera más amplia posible, utilizamos el concepto de padecimiento ${ }^{3}$ mediante el cual se busca comprender la forma como el individuo vive su aflicción que puede estar asociada o no a una enfermedad. En virtud de este andamiaje teórico, consideramos que mediante el análisis de las enunciaciones tanto de los temaxtianitl como de las mujeres que recibieron los tratamientos podríamos conocer la manera como funciona esta medicina y al mismo tiempo aproximarnos a diferentes aspectos de nuestra cultura que resultan paralelos al proceso salud-enfermedad.

\section{Método}

La investigación contó con dos etapas que abarcaron del año 1999 hasta 2003. La primera tuvo una orientación exploratoria donde la figura central fue un temaxtianitl, en la segunda la atención estuvo dirigida hacia algunas mujeres que acudieron con él. El objetivo de la primera fase fue describir y comprender los postulados y prácticas de la medicina practicada por un temaxtianitl, en particular sus nociones y técnicas dirigidas a la salud mental y emocional, mientras que el objetivo de la segunda fase fue comprender la forma en que intervinieron estas prácticas curativas en el tratamiento de problemas emocionales mediante las narraciones de las pacientes. Es importante aclarar que el temaxtianitl atiende tanto hombres como mujeres de todos los niveles socioeconómicos. Sin embargo, de acuerdo con nuestras observaciones y a decir del curandero, la mayor demanda proviene de mujeres de clase media, por lo que decidimos trabajar con este grupo. Esta observación coincide con la información presentada por Campos (1997) acerca del tipo de población que suele visitar a una curandera que también ejerce sus prácticas en Ciudad de México.

En las dos fases realizamos una aproximación etnográfica que se llevó a cabo en distintos escenarios de Ciudad de México, entre otros, ferias de salud, actos culturales, domicilios particulares de pacientes y la casa de curación. Todas las observaciones fueron registradas en un diario de campo que incluyó la serie de acontecimientos, comportamientos y conductas no verbales de los actores, así como las apreciaciones generales del observador. 


\section{Fase 1. La historia de vida de un temaxtianitl}

Para la primera fase decidimos realizar una historia de vida del vocero de la Tetzkatlipoka. Esperábamos que funcionara como prueba exploratoria y que nos ayudara a detectar nuevas líneas de investigación. Entre las ventajas que presenta la historia de vida para este tipo de estudio son que mediante la narrativa del individuo central y las de sujetos cercanos es posible tener un control de las variables que expliquen el comportamiento de éste dentro de su grupo primario, da respuesta a todas las eventuales preguntas e integra sus diferentes esferas sociales presentándolas de manera longitudinal como una trayectoria concreta (Pujadas 1992). Las razones por las cuales se realizó la historia de vida del vocero fueron las siguientes. Los miembros de la Tetzkatlipoka suelen asignar a una persona encargada de ser el portavoz de sus prácticas y costumbres frente a gente externa al grupo. La persona designada por el grupo se encontraba plenamente integrada a este medio simbólico-cultural lo cual la dotaba del conocimiento y total dominio del tema, además disponía de tiempo y reunía las condiciones óptimas para suministrar información (Taylor y Bodgan 1986). La información para realizar la historia de vida se obtuvo por medio de la entrevista en profundidad; las preguntas estuvieron dirigidas hacia la comprensión de las perspectivas que tenía el informante respecto a su vida tal como las expresaba en sus palabras. Para recopilar la información se elaboró una guía de entrevista con los siguientes temas claves: (1) Antecedentes familiares, (2) Historia de su formación dentro de esta tradición, (3) Participación dentro de la Tetzkatlipoka, (4) Caracterización de la tradición, (5) La salud mental y la Tetzkatlipoka y (6) Relación con otros servicios. Todos los encuentros fueron audiograbados y posteriormente transcritos en papel; en la transcripción se tomaron en cuenta las palabras y algunas características de la conversación (risa, pausas largas, gestos, entre otras). Una vez concluido este trabajo se inició el análisis del material obtenido mediante la técnica de categorización de significados propuesta por Kvale (1994).

En primer término se definieron categorías excluyentes que guardaron correspondencia con la guía de entrevista en donde se organizaron las unidades del material obtenido, por ejemplo, una de las categorías fue "formación para ser temaxtianitl". En un segundo paso, las categorías se subdividieron con base en un criterio de variabilidad, por ejemplo, la categoría mencionada se subdividió en "formación paterna" y "formación con médicos tradicionales", de esta manera se facilitó la descripción de las características más importantes del contenido y por ende la comprensión de cualquier fenómeno particular que hubiera ocurrido a lo largo de la entrevista.

\section{Fase 2. Entrevistas a cinco mujeres que recu- rrieron con los temaxtianitl}

Antes de iniciar la segunda fase solicitamos el consentimiento bajo información previa, tanto de los temaxtianitl para trabajar con los usuarios de sus servicios médicos como de las personas que participaron en calidad de informantes. El consentimiento bajo información previa consistió en explicarles los objetivos de la investigación, hacerles saber cuáles instituciones y personas estaban involucradas en el estudio, la manera como se estaba trabajando y cómo se iba a utilizar la información obtenida.

En primera instancia se realizaron pláticas informales con los usuarios que acudieron a la casa de curación con el objetivo de detectar quiénes eran los que recibían tratamiento para algún trastorno mental o emocional y bajo este criterio elegimos a cinco mujeres. También utilizamos la entrevista en profundidad que se basó en una guía que tuvo los siguientes temas: (1) Formas de acceso a esta medicina, (2) Enfermedad, padecimiento o problema emocional que fue tratado por los médicos de la tetzkatlipokatl, (3) Prácticas y recursos utilizados con anterioridad o al mismo tiempo para tratar ese tipo de enfermedades y padecimientos, (4) Percepción de su experiencia con las técnicas y recursos de esta medicina así como de otros servicios, (5) Evolución de los padecimientos y/o enfermedades tratadas por estos médicos tradicionales y evaluación de los tratamientos.

En seguida mencionaremos algunas de las características de las participantes (los nombres son seudónimos). Iniciamos con Virginia, ella es la de mayor edad, en el momento de la entrevista tenía 58 años y residía en Ciudad de México. Ella estudió hasta el último grado del nivel secundaria y se dedicaba al hogar. Le seguían, por orden descendente de edad, Isabel y Bertha, ambas tenían 30 años; Bertha vivía en Ciudad de México, Isabel, originaria de Israel, se encontraba realizando un viaje largo dentro de la República Mexicana. Bertha había terminado sus estudios de licenciatura y trabajaba como mando 
medio en una empresa privada. Por su parte, Isabel había concluido el nivel medio superior y antes de emprender un viaje que le tomó un año se dedicaba a modelar y a cantar de manera semiprofesional. Les seguía Luisa, con 29 años; había concluido sus estudios profesionales y trabajaba para una empresa privada donde ocupaba un nivel de mando medio. Finalmente, la más joven fue Gloria, de 25 años, ella también había completado el nivel de licenciatura y tenía trabajos temporales.

Todas las entrevistas fueron audiograbadas y transcritas en papel bajo los principios que mencionamos párrafos atrás. Se decidió estudiar cada entrevista mediante un modelo estructural propuesto por Labov y Waletzky (1967). Consistió en reconocer las unidades narrativas contenidas en cada entrevista y después organizarlas dentro de diferentes categorías analíticas. El primer paso fue segmentar unidades narrativas en los siguientes componentes: orientación, nudo o complejización, evaluación, solución y coda; no todas las narraciones presentaron cada uno de tales componentes pues la reunión de todos estos conforman un prototipo. El contenido de cada una de las narraciones fue examinado a la luz de las categorías (construidas de acuerdo con los temas de la entrevista) y cuando fue necesario se integró con el resto de la entrevista. De esta manera se mantuvieron unidades discursivas consistentes que pudieron someterse a comparación con otras unidades de la misma entrevista o con unidades pertenecientes a otras entrevistas sin que se perdiera el contexto o el sentido de lo expresado por las informantes.

\section{La Wewepahtli y sus Practicantes}

\section{Los que portan el sistema o temaxtianitl}

En el movimiento denominado "mexicanidad" convergen diferentes grupos, uno de ellos es el Tetzkatlipoka. Resulta común entre los líderes de este tipo de agrupaciones presumir, sin pruebas de esto, de una especie de continuidad sanguínea entre ellos y algunas figuras precolombinas importantes como Cuauhtémoc, Nezahualcóyotl, entre otros (Odena 1984; Tlakaelel et al. 1992). Tal idea de continuidad le sirve de argumento para ser reconocidos por su comunidad como los legítimos custodios de un legado que consiste en conocimientos astronómicos, médicos, filosóficos e históricos. En la Tetzkatlipoka hay un par de líderes que aseguran guardar tanto este tipo de lazos de parentesco como la función correspondiente de custodios, debido a lo cual ostentan los estatus más altos dentro del grupo.

Su organización está basada en diferentes jerarquías y en la distinción de sexos, ambas suponen una división de tareas y privilegios. Su práctica médica es sensible a este último aspecto pues los hombres tienen primacía para curar sobre las mujeres, lo que genera una especie de monopolio de la información por parte de los varones. La preparación de un futuro temaxtianitl requiere, además del dominio técnico (herbolaria, temascales, etcétera), una alta competencia para aplicar en la vida cotidiana el calendario ritual, los significados de las metáforas contenidas en el lenguaje pictórico de los códices $\mathrm{y}$ esculturas precolombinas.

Los postulados que fundamentan estos saberes conforman una especie de sistema basado en diversas dualidades, frío-caliente, eléctrico-magnético, día-noche, que se encuentran sintetizadas en el símbolo conocido como el nahui ollin, en español, cuatro movimiento (la figura es la de una suástica o cruz). Con la palabra temaxtianitl se señala un grado alcanzado, son las personas que debido a sus conocimientos y total dominio de este sistema lo pueden llevar a la práctica. Durante la investigación conocimos a cinco hombres con el cargo de temaxtianitl y dos mujeres que "tenían permiso de curar". La preparación que un hombre necesita para poder ostentar el cargo de temaxtianitl puede llevar varios años. El aprendizaje consiste en la observación y la práctica que se adquiere ayudando a otros temaxtianitl o a diferentes médicos tradicionales, también es necesario acumular experiencias en la realización de ceremonias rituales. Es un aprendizaje de orden empírico, aunque muchos aprendices echan mano de bibliografía especializada, sobre todo en la cosmovisión mexica, y no falta quien complemente su entrenamiento con el apoyo de cursos en los que aprende acerca de otras medicinas (china, hindú).

\section{La casa de la curación. Acudir a la medicina de la Tetzkatlipoka}

El vocero de la Tetzkatlipoka y temaxtianitl narró que en 1991 el grupo abrió al público una casa de servicio llamada "Centro de desarrollo cultural Tetzkatlipoka kalpulli", en este lugar realizaban sus prácticas médicas (aunque solían acudir a otros 
espacios como domicilios particulares, ferias y plazas públicas). El lugar se cerró en el 2003 pero los temaxtianitl continuaron con el servicio de medicina en distintos lugares de la ciudad, en otros estados de la República Mexicana y en Estados Unidos de América.

Para asistir al servicio médico los usuarios debían hacer una cita, se les pedía asistir con cuatro horas de ayuno y que llevaran una sábana y aceite. De acuerdo con el vocero, durante los primeros años el pago era voluntario, el usuario decidía si remuneraba o no al terapeuta, entonces había personas que pagaban con dinero ${ }^{4} \mathrm{y}$ otras que lo hacían en especie (bolsas de arroz, dulces, comida, etcétera). Solamente la medicina (compuestos herbolarios) tenía un costo fijo de cincuenta pesos. Cuando se trató de estipular un costo fijo por cada servicio, los propios médicos terminaban por incumplirlo debido a los escasos recursos económicos de algunos usuarios así que la remuneración terminaba siendo pactada entre médico y usuario.

\section{Equilibrio-desequilibrio en la medicina wewepahtli}

El vocero del grupo explicó que la salud no es lo mismo para todas las personas, en lugar de "salud" ellos hablaban más bien de equilibrio, pero si se les pedía una definición de salud lo hacían en los siguientes términos: la salud es "la relación que [el ser humano] tiene a nivel familiar, comunal y con el Universo, incluyendo a todos los elementos que estén a su alrededor", es decir, trasciende el aspecto individual. Por eso los temaxtianitl usualmente indagaban sobre el medio familiar, laboral, la historia clínica y personal del usuario, decían que era necesario conocer todos estos aspectos para atenderlo de manera adecuada.

Entre las prescripciones generales para mantener el equilibrio mencionaban que una persona debería beber la cantidad adecuada de agua, consumir los alimentos en determinadas proporciones, con cierta frecuencia y aquellos convenientes para su organismo, además debería comportarse de manera apropiada con otras formas de vida como personas, animales, vegetales y minerales para que éstas no perdieran el equilibrio por su causa, también se debería cuidar la manifestación de las emociones y sentimientos de tal manera que no se guardara alguno sin expresar o que al expresarlo no se lastimara a otra forma de vida.
En contraparte, la enfermedad o desequilibrio surgiría cuando las personas perdieran el balance entre esos componentes corporales, sociales y ambientales que contribuyen con igual grado de importancia en el equilibrio. Es posible que las personas nunca alcancen un equilibrio completo porque se encuentran sujetas a un entramado de determinantes económicas, sociales y culturales, entonces la tarea debería ser procurar acercarse al equilibrio. Para la wewepahtli el componente más delicado son las emociones, solían referirse a la vulnerabilidad de las emociones con la siguiente frase: "las emociones son la boca de las enfermedades". Según los temaxtianitl, las emociones constituyen el componente que con mayor frecuencia puede desequilibrarse y traer consigo el desequilibrio de otros componentes, por ejemplo el físico, así que un dolor de estómago, la tensión en el cuello o inclusive una parálisis de algún miembro puede tener su causa en el desequilibrio de las emociones.

\section{La técnica diagnóstica y curativa, apapaxtli tlawayotl maihpahtli}

Lo mencionamos solamente como apapaxtli, pero su nombre completo es apapaxtli tlawayotl maihpahtli y significa "ablandar lo que genera el corazón de la gente con las manos que curan", consiste en la manipulación y digitopresión corporal. En palabras del vocero, mediante esta técnica es posible tocar y acomodar los músculos, huesos, tendones y articulaciones, además de estimular los sistemas linfático y nervioso. Como recurso diagnóstico le permite al médico explorar el cuerpo y reconocer las zonas que pudieran tener algún funcionamiento inadecuado, por ejemplo, alguna desviación ósea, una deficiencia en cualquier órgano o alguna emoción contenida. Como tratamiento, sirve para corregir algunos de los malestares de manera directa, por ejemplo, si se presenta alguna contractura muscular o si se requiere estimular la vía respiratoria, hay ocasiones en que los temaxtianitl aplican al mismo tiempo su aliento en forma de exhalaciones y soplidos, cantos o silbidos.

Las personas que recibían el apapaxtli debían permanecer acostadas, sin ropa y cubiertas solamente por una sábana; para facilitar la manipulación del cuerpo los médicos utilizaban aceite e invariablemente seguían un orden específico con cada sección del cuerpo. Debido a la necesidad de permanecer 
totalmente desnudas y considerando que podría resultar incómodo para la gente, los temaxtianitl solían preguntar a las personas que asistían por primera vez qué sabían acerca de su medicina y antes de pasar al cuarto de curaciones el médico les explicaba en qué consistía el apapaxtli. Una vez que el médico se encontraba aplicando el apapaxtli, solía hacer observaciones o preguntas al usuario, quien podía limitarse a responder, entablar una conversación con el médico o guardar silencio.

La duración de los tratamientos oscilaba entre cuarenta y noventa minutos; las reacciones que se pudieron apreciar en diferentes personas fueron variadas: risa, llanto, quejidos, gritos, agitación, movimientos involuntarios de piernas, brazos y relajación. Cuando se les preguntó a los temaxtianitl por las causas de estas reacciones señalaron que los motivos dependían de los desequilibrios presentes en cada caso, pero de manera general todas las reacciones formaban parte de las emociones contenidas y mediante el apapaxtli podían empezar a removerlas. El vocero explicaba el funcionamiento del apapaxtli mediante una metáfora, decía que las emociones son como ríos que fluyen, cuando dejan de fluir, el agua se estanca y empieza a oler mal. Mediante el apapaxtli ayudaban a que estos "ríos" fluyeran nuevamente.

Un comentario común por parte de los usuarios acerca de este tratamiento fue que dolía mucho, pero los temaxtianitl decían que el apapaxtli no causaba dolor, sino que eran las tristezas, enojos, alegrías que la persona guardaba sin expresar lo que provocaba dolor en el momento de recibir el apapaxtli. Cabe decir que no siempre e invariablemente el apapaxtli resultaba doloroso; conforme avanzaba el tratamiento, si la persona lograba alcanzar cierto grado de equilibrio y recibía con frecuencia el apapaxtli, el dolor diminuía y junto con él las reacciones más aparatosas. Esto se debía a que con cada sesión se removían emociones y se corregían otros desequilibrios. Como apoyo a este procedimiento, los temaxtianitl solían trabajar en la "modificación de hábitos y costumbres", se trataba de dar recomendaciones dirigidas a mejorar la calidad de la respiración, el descanso, la hidratación y la alimentación de las personas. La herbolaria tenía un lugar importante en esta medicina, la utilizaban para preparar infusiones, agua de uso y ramos que colocan sobre el cuerpo.

\section{Las Mujeres que Recibieron el Apapaxtli}

\section{¿Cómo se enteraron de este servicio médico?}

La forma más extendida de anunciarse entre los especialistas en medicina "tradicional" mexicana continúa siendo la recomendación de persona a persona, así que las redes sociales de las entrevistadas resultaron de suma importancia. Gloria y Virginia se enteraron por medio de sus hermanas, otras dos mujeres supieron mediante sus amigas, mientras que una de ellas recibió la recomendación por parte de un médico homeópata. En el momento de establecer contacto con alguno de los temaxtianitl tres de las entrevistadas afirmaron que la información con la cual contaban era que se trataba de masajes, las demás dijeron que sólo sabían que ellos eran curanderos. En seguida Luisa narra la experiencia cuando por vez primera llegó a la casa donde fue atendida por uno de los temaxtianitl que, para guardar su anonimato, hemos designado como $\mathrm{E}$. Luisa hace alusión al momento en que le dan la información y de la manera como se debe recibir el apapaxtli, sin ropa alguna.

Este, bueno llegamos la casa de E. [el temaxtianitl] y nos dio una hoja donde venía toda la explicación de la cultura indígena que siguen, en la que creen, [nos explicó] cómo era el proceso de los masajes; [dijo] que nos daba un tipo de cantos, [dijo] que no era falta de respeto. Una cosa que nos llamó la atención fue que si sentíamos que nos faltaba al respeto en cierto momento, que debíamos de romper su, bueno su ídolo [una figura hecha de barro], el ídolo que tiene porque en dado caso es una ofensa y que no podía practicarlo más ¿no? Ese fue el primer contacto aparte de que tenía a un chavo adentro, masajeándolo que andaba gritando.

\section{Los motivos de la visita, los diagnósticos y los padecimientos}

Solamente una de las entrevistadas acudió por una demanda de atención específica, Virginia. A ella le dolía una pierna de manera recurrente y a decir del temaxtianitl que la trató, el dolor se debía a que un nervio se encontraba sumamente oprimido por una vértebra. Le preguntamos a Virginia a qué 
le atribuía su dolor y respondió que esa tensión seguramente era el resultado del estrés; explicó que suele ser presa fácil de las preocupaciones y tiene una sensibilidad especial ante los problemas de su familia. Virginia le dijo lo mismo al temaxtianitl y éste le hizo algunas preguntas acerca de sus problemas y las situaciones que le causaban estrés. Durante y después del apapaxtli, el temaxtianitl sostuvo varias conversaciones con ella, le prescribió una serie de ejercicios para corregir las posturas corporales con el objetivo de liberar el nervio de la opresión de las vértebras y le recomendó otros ejercicios para deshacerse del estrés.

En este caso el motivo de la visita, un dolor físico que puede ser "tocado" por los temaxtianitl y que incluso podría ser medido bajo otros modelos médicos, fue atendido de acuerdo con los postulados de la wewepahtli. Al mismo tiempo, el problema de orden existencial de Virginia, su manera de ser o estar en el mundo, también encontró un espacio y un tiempo para ser atendido. La explicación que Virginia tenía sobre su dolor no fue contradicha ni puesta en duda por el médico. Sólo que dependerá totalmente de ella el éxito en modificar sus conductas e ideas que contribuyeron a desequilibrarla. Este es el estilo de atención de los temaxtianitl, con los siguientes casos explicaremos otras de sus características.

Luisa y Bertha acudieron por primera vez con los temaxtianitl porque querían relajarse con un masaje, pero desconocían qué tipo de masaje recibirían y tampoco sabían que formaba parte de la medicina "tradicional" mexicana. Isabel acudió con ellos porque buscaba una guía espiritual. En términos estrictos y desde el punto de vista de la medicina científica, tanto relajarse como la orientación espiritual cabrían de manera bastante forzada dentro del campo médico, más bien corresponderían a los órdenes del descanso-esparcimiento y al de la religión. Sin embargo, para los temaxtianitl ambas demandas son válidas y cuentan con recursos para atenderlas.

Para Bertha, Luisa e Isabel sus demandas definitivamente corresponden al campo de la salud, pero sabían que para atenderlas debían recurrir a servicios o realizar actividades que suelen guardar escasa relación con la medicina científica.

$\mathrm{Al}$ respecto cabe señalar que las cinco entrevistadas recurrieron a este tipo de servicios y actividades; Gloria, Isabel, Luisa y Bertha coincidieron en la práctica del yoga; Virginia e Isabel estuvieron en contacto con el reiki, la primera lo estudió mientras que la segunda lo recibió como tratamiento. A Luisa y Bertha les leyeron las cartas (tarot). Isabel practicó el budismo tibetano, hizo meditaciones y retiros. Bertha suele leer y llevar a la práctica la metafísica en su vida cotidiana. Luisa asistió con un santero y recibió limpias con huevo y yerbas. Cuando indagamos los motivos que tuvieron para asistir a cada una de estas prácticas notamos que destacaron los problemas de índole emocional.

Continuando con los motivos que tuvieron para asistir con los temaxtianitl, le preguntamos a Luisa por qué consideraba que necesitaba relajarse con un masaje y comentó que en primera instancia tenía que lidiar con la tensión que le ocasionaba el ritmo diario (trabajo, escuela, familia, etc.); en seguida dijo que durante ese periodo se encontraba particularmente sensible debido al reciente fallecimiento de su padre que trajo consigo un reordenamiento de su vida. Relató que durante el primer tratamiento recibió el apapaxtli y comenzó un diálogo con el médico que le provocó una sensación de alivio porque platicó sobre sus dudas, temores y problemas.

Después de su primera sesión, el temaxtianitl le diagnosticó problemas de tabaquismo, sobrepeso y depresión. Como tratamientos fueron prescritos un preparado de yerbas, las indicaciones para modificar algunos hábitos y la recomendación de asistir a más sesiones de apapaxtli. Luisa siguió más o menos las indicaciones y acudió en varias ocasiones para recibir el masaje. Ella encontró que durante estas sesiones "se abría", podía descargar sus problemas y consideró que estas sesiones le ayudaban a relajarse.

Isabel tenía más de diez años tratando un estado depresivo que a veces cedía y otras se agudizaba, estaba segura de que la causa de sus depresiones era el miedo porque cuando le temía a algo se enfermaba. Ella mencionó que sentirse deprimida era normal porque la depresión formaba parte de su cultura y si a eso le sumaba varios sucesos de su vida personal, lo más lógico era deprimirse. Cuando acudió con los temaxtianitl buscaba una orientación para saber quién era ella y estar consigo misma, es decir, no consideraba que la depresión fuera el motivo para acudir con ellos. Uno de los temaxtianitl le propuso vivir un mes en una cabaña que estaba fuera de Ciudad de México con el objetivo de que aprendiera hábitos propios de la tradición Tetzkatlipoka en un ambiente de aislamiento. Durante la tercera semana 
se realizó la primera entrevista. Al preguntarle qué había sucedido durante este tiempo, mencionó lo siguiente,

Pienso en hace tres semanas, solamente hace tres semanas y hoy me siento en un nuevo comienzo. Y E. [temaxtianitl] lo preparó. Él sabía qué era lo que yo realmente necesitaba: a nadie. Lo que necesito es estar sin otra persona, lo que necesito es nada, yo necesito estar conmigo misma, necesito explorarme a mí misma y poder ver fácilmente mi verdadero rostro...él [temaxtianitl] solamente está para ayudar de muchas formas, está para ayudarte a encontrar tu propio maestro. No estoy diciendo que encontré a mi maestro en él, pero me mostró el primer paso. Es gracioso, es como hacer un viaje completo, muchas enseñanzas orientales hablan sobre esto, el Tao, el Zen, incluso el budismo.

En su caso, el tiempo que pasó con el temaxtianitl tendió a enfocarse más en el aprendizaje que en lo terapéutico, por ejemplo, las sesiones de apapaxtli no solamente tenían una intención curativa sino que mientras lo recibía el médico le enseñaba cómo aplicárselo ella misma. La modificación de hábitos la realizó bajo cierta supervisión. Cuando concluyó el mes de estancia en la cabaña comentó que pasó por algunos "momentos depresivos" y lo importante para ella fue que tuvo la oportunidad de enfrentarlos por sí misma.

Gloria llegó como acompañante de su hermana quien acudió para recibir atención por un problema en la rodilla. Gloria dijo que no buscaba ninguna forma de atención médica, no se sentía enferma ni tenía ningún problema. Sin embargo, cuando el médico les explicó en qué consistía el tratamiento también mencionó los diferentes problemas que podía atender y entre ellos se encontraban las fobias. Entonces Gloria recordó que desde niña le temía a los pájaros y que padecía de cólicos e irregularidades en su ciclo menstrual, por lo tanto decidió tomar el apapaxtli. Esa primera sesión la impresionó de manera profunda y decidió seguir con los tratamientos. La siguiente narración es la respuesta que dio cuando se le preguntó: ¿sabías qué tipo de tratamiento ibas a recibir con estos médicos?
No, la verdad yo no tenía idea. Mi hermana me dijo que se trataba de un masaje, de una terapia. Esa fue la terapia más fuerte (que he recibido), a mí me dolió muchísimo, fue muy larga. Entré a las cuatro y salí de ahí a las nueve de la noche; lloré, lloré mucho, o sea, fue muy emocional, además de que físicamente me dolió, fue muy emocional. Lloré mucho, me dormí, me volví a despertar, fue muy, muy fuerte. De ahí, tuve como un tiempo como de mucha inestabilidad emocional, como que se estaban ajustando cosas. Me impactó mucho, siento que hubo muchos cambios. Por eso regresé.

En esa ocasión el temaxtianitl le comentó que ella tendía a "guardarse las cosas", es decir, que no expresaba sus emociones con frecuencia. No le recomendó ningún otro tratamiento y le dijo que regresara cuando quisiera. La inestabilidad emocional a la que se refiere tiene que ver con una relación de pareja poco estable que había tenido por muchos años, dijo que después de esa primera sesión tuvo la determinación de romper este vínculo y que se sintió mejor. Gloria volvió a recibir el apapaxtli al cabo de cuatro meses y siguió un tratamiento herbolario para su padecimiento menstrual, además modificó algunos hábitos alimenticios y otras costumbres siguiendo los saberes de la wewepahtli.

La atmósfera ${ }^{5}$ curativa que construyeron los temaxtianitl para tratar a estas mujeres gozó de la suficiente amplitud para atender tanto sus padecimientos como los desequilibrios que les diagnosticaron. A veces los motivos por los que una persona asiste al servicio difieren de sus problemas y, al mismo tiempo, ambos difieren de la mirada médica, pero durante el acto curativo tanto lo que dice el usuario de sí mismo como las observaciones del temaxtianitl gozan de la misma importancia.

Para Isabel su problema no era estar deprimida sino que era la sensación de incapacidad para proporcionarse a sí misma las condiciones necesarias para estar en el mundo de la mejor manera posible, así que el acuerdo tácito que hizo el temaxtianitl con ella fue el de trabajar en este punto. Gloria fue tratada de manera similar, como para ella ni el miedo a los pájaros ni los trastornos menstruales eran problemas de salud, el médico no realizó ninguna prescripción al respecto hasta que ella decidió pedir ayuda específica. 
Notamos que los médicos tienden a expresar sus diagnósticos en términos técnicos antes que en términos teóricos. En lugar de afirmar que alguien se encuentra deshidratado dicen que necesita beber más agua, lo cual contribuye a que las explicaciones que ofrecen a las usuarias no sean traducidas o filtradas por un lenguaje demasiado especializado que pudiera resultar críptico para ellas. Al parecer el rasgo que distingue a esta práctica es una clase de "proximidad", entendiéndola como el resultado de las acciones recíprocas entre el usuario y el temaxtianitl. Gracias a cierta calidad en la proximidad se logra relajar y quizá hasta diluir la tensión entre el mundo interior y el mundo exterior del usuario.

En la topografía de estos mundos, las reflexiones que una persona elabora acerca de su comportamiento, sentimientos o las emociones más oscuras quedarían en el mundo interior, mientras que en el exterior cabrían las convenciones (o normas que regulan el comportamiento), las diversas situaciones (el trabajo, las muertes, la guerra) y los colectivos (la pareja, la familia, la nación). Por lo general todos los individuos invertimos buena parte de nuestra energía y tiempo evitando que los dos mundos colisionen, por ejemplo, cuando una persona se siente triste o desanimada pero debe mantener su atención en el trabajo, esa tristeza o desánimo deben pasar a un segundo plano, lo cual no significa que entren en "receso" ni mucho menos que desaparezcan; cabe la posibilidad que en virtud de una proxémica sistematizada las fronteras entre yo y los otros, el ser y el deber ser se suavicen y la tensión disminuya al menos momentáneamente. Dicha proximidad ocurriría durante el contacto y manipulación corporal en el apapaxtli.

\section{Las experiencias con el apapaxtli}

Las cinco mujeres recibieron el apapaxtli; recordemos que, además de ser un recurso diagnóstico, lo es también curativo, y de acuerdo con ellas esta curación puede ser muy dolorosa. Isabel describe su primera sesión.

¡Ah! Casi moría, fue la experiencia más dolorosa en mi vida. Físicamente fue el contacto más doloroso que he tenido en mi vida. Fue casi horrible pero después de este tratamiento, después de esta noche, fue una noche larga, fue realmente extraordinario para mí.
Como mencionamos párrafos atrás, se observó que las personas presentaron distintas reacciones durante el apapaxtli como enojo, sueño, dolor, etcétera. Nos pareció notorio la forma como algunas de las mujeres expresaron sus sensaciones, un ejemplo es lo que nos dijo Virginia:

Mira, no me dolió. Sentía como me iba subiendo calor. Calor por el cuerpo, por todo el cuerpo y sentía no una mano. ¿Qué te puedo decir? Como una bola que me envolvía toda la pierna, hacia arriba, pero donde sí me dolió mucho fue en la ingle. En la ingle y en la parte de la espalda. Ahí sí me dolió, pero en los brazos, sentía esa lumbre que me iba subiendo por todo el cuerpo. Pero no me sentía mal y no me dolía, no más ahí en esas partes fue donde me dolió y me dolió mucho, como si me hubiera metido un clavo y me lo hubiera sacado del otro lado.

Una de las ideas básicas de la wewepahtli es que en el universo existen un conjunto de energías que deben circular, estas energías se manifiestan en el agua, el aire, los ciclos de vida, todos ellos deben fluir con el objetivo de guardar el equilibrio. Como las emociones son una forma de energía de igual manera deben mantenerse en constante movimiento. Los diferentes estados anímicos y reacciones que experimentaron las mujeres mediante el apapaxtli fueron la expresión de las emociones que por diversas causas han quedado estancadas y que los médicos ayudaron en primera instancia a "ablandar" para que en seguida pudieran fluir.

En palabras de las entrevistadas, el masaje tiene "matices psicológicos" que al parecer no esperaban porque para ellas una manipulación física debería tener consecuencias físicas, no emocionales. No obstante, a las entrevistadas les pareció que esta característica del apapaxtli las benefició y en términos generales calificaron de manera positiva los recursos y métodos de la wewepahtli. Bertha dijo que el masaje le dio mucha energía y ánimo para realizar sus tareas; este estado anímico le duró alrededor de un mes. A Luisa las pláticas le ayudaron a tranquilizarse y a ser consciente acerca de sus posturas corporales al caminar, permanecer sentada, etcétera. Para Isabel el mes que pasó en la cabaña le ayudó a actualizar y confirmar algunas enseñanzas ideológico-prácticas que le suelen ayudar 
a enfrentar sus momentos depresivos. Gloria dijo que tanto las infusiones, el cambio de hábitos y las sesiones de apapaxtli le corrigieron los trastornos menstruales y atenuaron en mucho su temor a los pájaros. Virginia afirmó que el malestar de la pierna mejoró bastante y que la modificación de hábitos le ayudó a mejorar su digestión y a limpiar la piel de su rostro (tenía una verruga).

\section{Algunas Consideraciones sobre la Wewepahtli}

Las dudas, preocupaciones y dolores que manifestaron las entrevistadas son la parte visible de la noción teórica denominada padecimiento; el lado oscuro o al menos poco visible es el cómo una pierna o un útero se convierten en centros dolorosos o cómo la inseguridad laboral, la ausencia de la pareja devienen en preocupaciones. Este tipo de estados constituyen "la carne" del proceso salud-enfermedad y es precisamente frente a los cuales el dominio médico corre el riesgo de verse desbordado.

Pensando en esta clase de debilidad, consideramos que la aportación más puntual de la wewepahtli es su estilo de atención, puesto que los temaxtianitl son sensibles a las dimensiones social, cultural, biológica y psíquica que forman parte de la condición del malestar de la persona.

Otra cuestión notable es el modelo de ser humano que propone esta medicina y en el cual se destaca el cuidado de los vínculos de cualquier persona consigo misma (sin la dicotomía soma-psique), con otros seres humanos y con el universo. Esta imagen de hombre confronta la científica; para darnos una idea al respecto tomamos las palabras de Sloterdijk (2003:124), quien señala que entre los siglos XVI y XVII junto con la ciencia de la disección se fue extendiendo cierta imagen del ser humano en la que éstos eran "ante todo y después de todo cuerpos aislados, que no remiten a nada: cuerpos que existen en unidad originaria funcional (máquina compuesta por órganos) y en individualidad organísmica para ser integrados después y en segunda línea en grupos sociales". La oportunidad para poner en práctica un modelo distinto ocurre durante el apapaxtli, en primer término, porque lo somático y lo psíquico no permanecen disociados (o se atenúa la distancia entre ambos) y en seguida debido a la calidad de la proximidad entre el médico y el usuario que ya se mencionó con anterioridad.
Consideramos que la experiencia con el apapaxtli puede ser más bien efímera y por lo tanto sus efectos no pueden ser ni prolongados ni mucho menos permanentes. Los individuos se encuentran expuestos de manera cotidiana a diferentes situaciones que pueden ocasionar desequilibrios, entre éstos la acumulación de emociones que no se expresan. De tal forma que para mantener un adecuado nivel emocional se debería "limpiar" continuamente este nivel, es decir, acudir periódicamente a recibir un apapaxtli.

Una de las grandes limitaciones de los métodos de la wewepahtli se hace evidente en la técnica de "modificación de hábitos" porque requiere de un programa educativo completo (recordemos que se trata de prescripciones respecto a la forma de respirar, descansar, hidratarse y comer) y el contenido de este programa suele oponerse a los estilos de vida urbanos. La mayoría de los habitantes de las grandes urbes, como Ciudad de México, tienen un ritmo de vida acelerado y estresante que les impide contar con el tiempo necesario para comer a horas adecuadas, descansar, hacer deporte, etc.

Nos gustaría subrayar el carácter paradójico de un objeto cultural como la wewepahtli y que podríamos encontrar en otras medicinas. Aunque nació y está organizada bajo los supuestos de la vida urbana contemporánea, gran parte de su éxito se debe a que ofrece un espacio y tiempo que contradice o escapa de estos supuestos. Para concluir citamos a Berman (2004:35), quien afirma que hoy en día "más que una nueva espiritualidad se necesita una nueva corporalidad", tal vez una capacitada para des-incorporar de manera voluntaria los órdenes sociales y culturales que dañen los vínculos que la conforman.

Agradecimientos: Nuestro más sincero agradecimiento por su colaboración a cada uno de los miembros de la Continua Tradición Tetzkatlipoka, así como a las cinco mujeres que de manera generosa aceptaron narrar sus experiencias.

Nuestro reconocimiento al Instituto Nacional de Psiquiatría y al Centro de Investigaciones y Estudios Superiores en Antropología Social por las facilidades otorgadas para la realización del estudio. Agradecemos a los evaluadores por sus acertadas sugerencias y comentarios que permitieron enriquecer este documento. 


\section{Referencias Citadas}

Anders, F.

1983 El Libro de Tezcatlipoca, Señor del Tiempo. Fondo de

Cultura Económica, México.

Augé, M. y C. Herzlich, editores

1994 The Meaning of Illness: Anthropology, History and

Sociology of Illness (Social Order). Routledge, London.

Berman, M.

2004 Historia de la Conciencia. De la Paradoja al Complejo de Autoridad Sagrada. Cuatro Vientos, Chile.

Campos, R.

1997 Nosotros los Curanderos. Nueva Imagen, México.

Hodge, R. y G. Kress

1993 Language as Ideology. Routledge, London.

Kleinman, A.

1980 Patients and Healers in the Context of Culture: An

Exploring of the Borderland Between Anthropology, Medicine and Psychiatry. University of California Press, Los Angeles.

Kvale, S.

1994 Methods of Analysis. Harwood Academic Publishers GmbH, Luxembourg.

Labov, W. y J. Waletzky

1967 Narrative analysis: Oral versions of personal experience.

En Essays on the Verbal and Visual Arts, editado por J. Helm, pp. 12-44. University of Washington Press, Seattle.

Lozoya, $\mathrm{X}$.

1999 La medicina tradicional hoy. Ponencia presentada en Ciclo de Medicina Tradicional Realizada en el Marco de la Exposición Los Mayas en el Antiguo Colegio de San Ildefonso, México.

Luz, M.

1999 Cultura contemporánea y medicinas alternativas: nuevos paradigmas en salud al finalizar el siglo XX. En Salud,
Cambio Social y Política. Perspectiva desde América Latina, coordinado por M. Bronfman y R. Castro, pp. 383-401. EDAMEX, INSP, México.

Martínez, F.

$1995 \mathrm{El}$ hombre y su padecer, centro de una nueva medicina. Médico Moderno 195:11-86.

Mateos, $\mathrm{H}$.

1993 Los Dioses Creadores. Enciclopedia Gráfica del México Antiguo. Secretaría de Hacienda y Crédito Público, México.

Meza, A.

1997 Nuestro Ser Interno. Universidad de Michoacán de San Nicolás de Hidalgo, México.

Odena, L.

1984 Movimiento Confederado Restaurador de la Cultura de Anáhuac. Centro de Investigaciones y Estudios Superiores en Antropología Social. SEP, México.

Pujadas, J.

1992 El Método Biográfico. CIS, Madrid.

Sloterdijk, P.

2003 Esferas I. Burbujas. Microesferología. Siruela, Madrid.

Taylor, S y R. Bogdan

1986 Introducción a los Métodos Cualitativos de Investigación. Paidós, Barcelona.

Tlakaelel, I. Luengas, G. Zenzes y P. Heuzé

1992 Nahui Mitl. Cuatro Flechas. UAM-X y Tomo II, México.

Vargas, L.

1991 Una mirada antropológica a la enfermedad y el padecer. Gaceta Médica de México 127:3-5.

\section{Notas}

1 Todas las voces distintas del castellano provienen del náhuatl y se ha respetado tanto la escritura como el significado que utilizan las personas que fueron entrevistadas.

2 La figura de Tetzkatlipoka ocupa un lugar relevante en la cosmogonía nahua ya que pertenece al grupo de los dioses creadores (Mateos 1993). El nombre de Tetzkatlipoka está compuesto de Tezcatl que significa espejo y tlepuca compuesto a su vez de tletl "chispa" y puctli "humo" (Anders 1983). Esta divinidad está asociada con la capacidad de revivir los sucesos que han quedado registrados en la memoria, es el espejo en el cual se reflejan nuestras experiencias y emociones (Meza 1997).

3 Que es el conjunto de significaciones que tiene para el sujeto un hecho que le acontece y las consecuencias del mismo para él y su entorno social. El padecimiento es la visión subjetiva de la enfermedad, es el conjunto de repercusiones psíquicas y sociales de la enfermedad. Estas significaciones no son inherentes, sino que se construyen en articulación de cada individuo con los distintos conjuntos sociales, es decir, es un saber cotidiano popular, de tal manera que lo que en un grupo social es calificado como enfermedad incide para que una persona de ese grupo se sienta o se autocalifique como enfermo; también incide en la manera en que se valoran e interpretan las acciones realizadas por médicos, curanderos, grupos de autoayuda, etc. De igual forma, el mundo de la religión y de la magia que envuelve a ese grupo social harán que la enfermedad y la curación se interpreten de un modo determinado, rezar para encontrar el alivio a la tristeza o someterse a una limpia para acabar con la enfermedad (Kleinman 1980; Martínez 1995; Vargas 1991).

4 La cantidad de dinero también variaba, algunos usuarios pagaban entre ciento cincuenta pesos y doscientos. La conversión en dólares sería aproximadamente entre 15 y 20 dólares.

5 Con esta noción nos referimos a la disposición de ánimo, en términos fenomenológicos es la primera apertura del ser en el mundo. 
